\title{
Deprivation Selectively Modulates Brain Potentials to Food Pictures
}

\author{
Jessica Stockburger \\ University of Konstanz
}

\author{
Alfons O. Hamm \\ University of Greifswald
}

\author{
Almut I. Weike \\ University of Greifswald \\ Harald T. Schupp \\ University of Konstanz
}

\begin{abstract}
Event-related brain potentials (ERPs) were used to examine whether the processing of food pictures is selectively modulated by changes in the motivational state of the observer. Sixteen healthy male volunteers were tested twice 1 week apart, either after $24 \mathrm{hr}$ of food deprivation or after normal food intake. ERPs were measured while participants viewed appetitive food pictures as well as standard emotional and neutral control pictures. Results show that the ERPs to food pictures in a hungry, rather than satiated, state were associated with enlarged positive potentials over posterior sensor sites in a time window of 170-310 ms poststimulus. Minimum-norm analysis suggests the enhanced processing of food cues primarily in occipito-temporo-parietal regions. In contrast, processing of standard emotional and neutral pictures was not modulated by food deprivation. Considered from the perspective of motivated attention, the selective change of food cue processing may reflect a state-dependent change in stimulus salience.
\end{abstract}

Keywords: human eating behavior, food deprivation, motivation, visual attention, ERPs

In natural environments, selective attention effects are often regulated by motivational states (Derryberry \& Tucker, 1994). Attention can be captured according to the inherent motivational significance of external cues, or it can vary by the organism's current need state. The former route of attention direction has been extensively studied in the past decade, comparing motivationally significant cues with neutral stimuli (Bradley et al., 2003; Lang, Bradley, \& Cuthbert, 1997; Öhman, Flykt, \& Lundqvist, 2000; Schupp, Junghöfer, Weike, \& Hamm, 2003; Vuilleumier, 2005). In contrast, much less is known about the latter route: the impact of dynamically shifting internal motivational states. When related to current motivational needs, stimuli may reflexively capture attentional resources to facilitate efficient responding (Lang et al., 1997). Food deprivation provides a model system to explore the neural mechanisms of state-dependent shifts in the perception of need-related food stimuli.

An attention bias for food cues in hungry state has already been suggested in behavioral research. By studying food words in the

Jessica Stockburger and Harald T. Schupp, Department of Psychology, University of Konstanz, Konstanz, Germany; Almut I. Weike and Alfons O. Hamm, Department of Psychology, University of Greifswald, Greifswald, Germany.

This research was supported by grants from the German research foundation Schu1074/7-1 and Schu1074/11-1 to Harald Schupp. The authors thank Thomas Schaer for his great support with data acquisition.

Correspondence concerning this article should be addressed to Jessica Stockburger, Institute of Psychology, University of Konstanz, PO Box 36, 78457 Konstanz, Germany. E-mail: jessica.stockburger@uni-konstanz.de modified Stroop paradigm, it has been shown that food deprivation delayed the naming of the color of food words (Channon \& Hayward, 1990; Lavy \& van den Hout, 1993; but see reservations in meta-analyses, Dobson \& Dozois, 2004). Similarly, research with the dot probe paradigm revealed that the attention capture of food words was enhanced in a hungry state but not a satiated state (Mogg, Bradley, Hyare, \& Lee, 1998; Placanica, Faunce, \& Soames Job, 2002). A differential processing for food pictures in a hungry state in comparison with that in a satiated state has also been demonstrated by psychophysiological measures (e.g., Drobes et al., 2001; Mauler, Hamm, Weike, \& Tuschen-Caffier, 2006) More direct evidence that hunger increases selective attention to food stimuli has been obtained using neuroimaging measures. A functional magnetic resonance imaging (fMRI) study revealed that viewing food pictures in a hungry state enhanced the activity in visual-associative brain regions as well as in the amygdala, a structure known to be involved in the detection of motivational and emotional significant stimuli (LaBar et al., 2001; Sabatinelli, Bradley, Fitzsimmons, \& Lang, 2005). An important extension of these findings is to determine the temporal dynamics of deprivationinduced effects on food cue processing.

This study explores whether hunger already modulates the perceptual processing of food cues by using event-related brain potentials. To reveal the obligatory nature of deprivation effects, participants passively viewed the stimuli. Because selective-attention studies observed changes in stimulus perception primarily under conditions of high perceptual load (cf. Luck, Woodman, \& Vogel, 2000), pictures were presented as a rapid serial stream. A further aim of this study was to determine the specificity of state-dependent variation in motivational sig- 
nificance. The use of nonfood appetitive as well as aversive pictures allowed us to test whether deprivation specifically modulates the processing of food cues. Deprivation may influence cortical reactions to appetitive stimuli in general (food and pleasant) or to emotional stimuli regardless of hedonic valence (pleasant and unpleasant).

\section{Method}

\section{Participants}

Sixteen healthy right-handed male volunteers (mean age: 24.3 years, $S D=4.8$ ) from the University of Greifswald participated in this study. Women were excluded for the chosen two-session design because of possible confounds due to the menstrual cycle. The mean body mass index was 23.1 ( $S D=$ 2.2). Participants received $15 €$ upon participation in both experimental sessions.

\section{Stimulus Materials}

Thirty-two appetizing food pictures depicting principle meals and desserts were taken from commercially available cookbooks. Nonfood control pictures $(N=96)$ were selected from the International Affective Picture System (IAPS; Lang, Bradley, \& Cuthbert, 2005), including pleasant, neutral, and unpleasant contents. The stimuli were presented as a continuous stream without a perceivable interstimulus gap, with each picture shown for $333 \mathrm{~ms}$. Pictures were presented in random order, assuring that the categories were equally often preceded by each other category. To increase signal-to-noise ratio, we repeated the set 10 times. $^{1}$

\section{Procedure}

Participants first attended a screening session, in which they were informed about the requirements for implementing the food deprivation condition and the normal eating condition. Questions from the Diagnostisches Interview bei Psychischen Störungen (Diagnostical Interview for Mental Disorders; Margraf, Schneider, \& Ehlers, 1991) were administered, and eating habits were assessed by standardized eating scales (Inventar zum Essverhalten und Gewichtsproblemen; Diehl \& Staufenbiehl, 1994; including German adaptations from the Three-Factor Eating Questionnaire (TFEQ), Stunkard \& Messick, 1985). Participants with eating disorders, food allergies, vegetarian diet, or other patterns of selective eating were excluded. Further exclusion criteria were current use of medication, psychiatric or neurological illness, and diabetes mellitus. Written informed consent approved by the Review Board of the University of Greifswald was provided by the participants.

Each participant was tested on 2 days, 1 week apart, either in a food-deprived state or a satiated state. The order of these experimental sessions was counterbalanced. Testing always occurred after lunch time to control for variations in circadian rhythm. Before the food deprivation session, participants were asked to refrain from eating anything and drinking sweetened beverages for $24 \mathrm{hr}$. Instructions for the satiated session required participants to follow their normal eating habits and to have a regular lunch. Before both testing sessions, participants were requested to record their food and fluid intake and to abstain from drinking alcohol. Participants were informed that they would have to give a urine sample prior to each session and were thus encouraged to disclose any food that they had eaten (used as a bogus pipeline; cf. Drobes et al., 2001). All participants (except 1 who was excluded from the final sample) reported that they had complied with these requirements.

On each session, participants rated their subjective feeling of hunger and appetite. For the EEG measurement, participants were instructed to passively view the picture stream. Afterward, pictures had to be evaluated on a 9-point Likert scale on the dimensions of valence and arousal (cf. Bradley \& Lang, 1994).

\section{Data Collection, Reduction, and Analysis}

\section{Subjective Ratings}

Hunger and appetite ratings were separately analyzed by using $t$ tests that compared hungry versus satiated states. Picture ratings were analyzed for valence and arousal with analyses of variance (ANOVA)s with repeated measurement on the factors of State (hungry and satiated) and Picture Category (food, pleasant, neutral, and unpleasant).

\section{Event-Related Brain Potentials (ERPs)}

Electrophysiological data were collected from the scalp by means of a 129-lead geodesic sensor net (Electrical Geodesics, Inc. [EGI], Eugene, OR). The EEG was recorded continuously with a sampling rate of $250 \mathrm{~Hz}$, with the vertex sensor as reference electrode, and was filtered online from .01 to $100 \mathrm{~Hz}$ by using Netstation acquisition software and EGI amplifiers. Impedances were kept below $30 \mathrm{k} \Omega$, as is recommended for this type of amplifier by EGI guidelines. Off-line analyses were performed using ElectroMagnetic EncephaloGraphy Software (EMEGS) (Junghöfer \& Peyk, 2004), including low-pass filtering at $30 \mathrm{~Hz}$, artifact detection, sensor interpolation, baseline correction, and conversion to an average reference (Junghöfer, Elbert, Tucker, \& Rockstroh, 2000). Separate averages were calculated for food and nonfood control pictures in the two sessions for each sensor and participant.

To uncover effects of motivational state on picture processing, a first stream of analysis was based on single-sensor waveform analyses. In these analyses, ANOVAs containing the withinsubjects factors of State (hungry and satiated) and Category (food, pleasant, neutral, and unpleasant) were calculated for each time point after picture onset separately for each individual sensor. To guard against chance findings, we considered significant effects meaningful only when the effects were observed for at least six continuous data points $(24 \mathrm{~ms})$ and two neighboring sensors (cf. Sabbagh \& Taylor, 2000). Results revealed a significant interaction of State and Category in the time window from 170 to $310 \mathrm{~ms}$ poststimulus over posterior sensor sites. These effects were mirrored over centrofrontal sites, that is, with opposite polarity.

In a second stream of analysis, these effects were explored by conventional ANOVA analyses on the basis of mean activity in selected sensor clusters and time windows. For brevity, only

\footnotetext{
${ }^{1}$ Previous studies already showed that the processing of emotional stimuli does not vary as a function of stimulus novelty (cf. Schupp et al., 2006).
} 
posterior sensor regions are reported. Mean amplitudes from representative posterior sensor clusters, identified by both visual inspection and waveform analyses, were averaged for three time intervals ranging from 170 to $210 \mathrm{~ms}$, from 220 to $260 \mathrm{~ms}$, and from 270 to $310 \mathrm{~ms}^{2}$ Data were entered into a four-factorial ANOVA including the within-subjects factors Time (three intervals: $170-210,220-260$, and 270-310), State (hungry and satiated), Picture Category (food, pleasant, neutral, and unpleasant), and Laterality (left and right).

Additional control analyses concerning potential carryover effects in the rapid serial presentation paradigm indicated that the deprivation effects were not modulated as a function of the valence of the preceding picture: State $\times$ Preceding Picture Category, $F(2,30)=1.8, n s$.

L2 minimum-norm analysis. L2 minimum-norm (MN) solutions were calculated to provide neural-source estimations for the differential processing of food stimuli as a function of motivational state. Calculations were based on a spherical four-shell isotropic volume conductor head model with 3 (radial, azimuthal, and polar direction) $\times 197$ evenly and spherically distributed dipoles as a source model. A source shell radius of $6 \mathrm{~cm}$ was chosen as a trade-off between depth sensitivity and spatial resolution (Hauk, 2004).

Replication of selective emotion processing. To replicate previous findings of selective emotion processing, and to explore unspecific effects of food deprivation on emotion processing, we scored the early posterior negativity (EPN) in bilateral temporooccipital clusters in a time window from 200 to $300 \mathrm{~ms}$ (cf. Schupp et al., 2007). Data were entered into an ANOVA, including State, Valence (pleasant, neutral, and unpleasant), and Laterality.

\section{Results}

\section{Behavioral Data}

\section{Hunger and Appetite}

As was expected, subjective feelings of hunger and appetite were increased in deprived states in comparison with satiated states, $t \mathrm{~s}(1$, $15)=17.9$ and 13.4, $p<.001$, respectively (see Table 1).

Table 1

Subjective Ratings of Hunger and Appetite and Picture Evaluation on Dimensions of Valence and Arousal for Food and Nonfood Control Picture

\begin{tabular}{lcc}
\hline \multicolumn{1}{c}{ Variable } & Satiated & Hungry \\
\hline Hunger $^{\mathrm{a}}$ & $2.85(1.09)$ & $8.00(0.68)^{*}$ \\
Appetite $^{\mathrm{b}}$ & $1.44(1.81)$ & $8.41(1.62)^{*}$ \\
Valence $^{\mathrm{c}}$ & & \\
Food $^{\text {Pleasant }}$ & $6.36(1.10)$ & $7.53(0.90)^{*}$ \\
Neutral & $7.18(0.87)$ & $7.20(0.91)$ \\
$\quad$ Unpleasant & $5.43(0.78)$ & $5.47(0.62)$ \\
Arousal & $2.92(0.61)$ & $2.93(0.87)$ \\
$\quad$ Food & & \\
Pleasant & $2.75(1.50)$ & $4.39(1.78)^{*}$ \\
Neutral & $4.96(1.74)$ & $2.86(1.57)$ \\
$\quad$ Unpleasant & $2.47(1.25)$ & $5.54(1.36)$ \\
\hline
\end{tabular}

Note. Standard deviations are in parentheses.

a Score range from 1 to 9 , with higher scores indicating stronger feelings of hunger. ${ }^{\mathrm{b}}$ Score range from 0 to 10 , with higher scores indicating stronger feelings of appetite. ${ }^{\mathrm{c}}$ Score range from 1 (very unpleasant) to 9 (very pleasant). ${ }^{\mathrm{d}}$ Score range from 1 (very calm) to 9 (very exciting).

* Significant difference between satiated and hungry state, $p<.01$.

\section{Picture Evaluation}

When food-deprived, participants rated the food pictures as significantly more pleasant, $t(15)=5.0, p<.001$, and arousing, $t(15)=4.0, p=.001$, in comparison with the satiated state. Specificity of deprivation effects for food stimuli was indicated by significant interactions of State $\times$ Picture Category for valence and arousal ratings, $F \mathrm{~s}(3,45)>10.8, p<.001$. Valence and arousal ratings of the nonfood emotional control pictures were not influenced by deprivation, State: $F \mathrm{~s}(1,15)<1$; State $\times$ Picture Category: $F \mathrm{~s}(2,30)<1$.

As in previous research, ratings of pleasant, neutral, and unpleasant control pictures significantly varied in valence, $F(2$, $30)=161.7, p<.001$. Furthermore, pleasant and unpleasant pictures were evaluated as more arousing than were neutral stimuli, $F(2,30)=74.6, p<.001$ (see Table 1$)$.

\section{ERP Data}

As is illustrated in Figures 1A and 1B, the ERPs to food pictures in a hungry, rather than a satiated, state were associated with enlarged positive potentials over posterior sensor sites in a time window from 170 to $310 \mathrm{~ms}$ poststimulus. This deprivation effect was specifically observed for the processing of food cues. There were no significant differences in the processing of nonfood control pictures between the hungry state and the satiated state (cf. Figure 1C). Statistical analyses supported these observations. A significant interaction of State and Picture Category, $F(3,45)=$ $4.2, p<.05$, was obtained, which was followed up by separate analyses of food and nonfood control pictures.

\section{Food Cues}

Food cues elicited an increased posterior positivity in a hungry state in comparison with a satiated state, $F(1,15)=4.6, p<.05$. The interaction of Time $\times$ State was not significant, $F(2,30)<1$. For exploratory purposes, the time intervals were analyzed separately. As is illustrated in Figures $1 \mathrm{~B}$ and $1 \mathrm{C}$, the effect of food deprivation appeared most pronounced in the first and last time intervals, $F s(1,15)=5.3$ and $7.3, p<.05$, respectively, while it attenuated in the second interval, $F(1,15)=1.6$, ns. While laterality effects are suggested in Figure 1, neither the main effect of Laterality, $F(1,15)<1$, nor higher-order interactions of State $\times$ Laterality, $F(1,15)=2.0, n s$, or State $\times$ Laterality $\times$ Time, $F(1$, $15)<1$, were significant.

\section{Nonfood Control Pictures}

The processing of the nonfood control pictures was not influenced by deprivation for pleasant, neutral, and unpleasant pictures, $F_{\mathrm{s}}(1,15)<1.9$, $n s$, respectively. No higher-order interactions, including State $\times$ Time, reached significance, $F \mathrm{~s}<1$.

\footnotetext{
${ }^{2}$ As the topographical focus of the difference varied over time, the clusters for the three intervals were adjusted (EGI sensor numbers of the first cluster: $31,32,37,38,43,48,51,52,53,54,59,60,61,66,67,72$ [left], 77, 78, 79, 80, 81, 85, 86, 87, 88, 92, 93, 94, 98, 99, 105, 106 [right]; second cluster: $32,38,43,52,53,54,61$ [left]; 79, 80, 81, 87, 88, 93, 94 [right]; third cluster: 7, 31, 32, 37, 38, 43, 48, 52, 53, 54, 60, 61, 67 (left) $78,79,80,81,86,87,88,93,94,99,105,106,107$ [right]).
} 

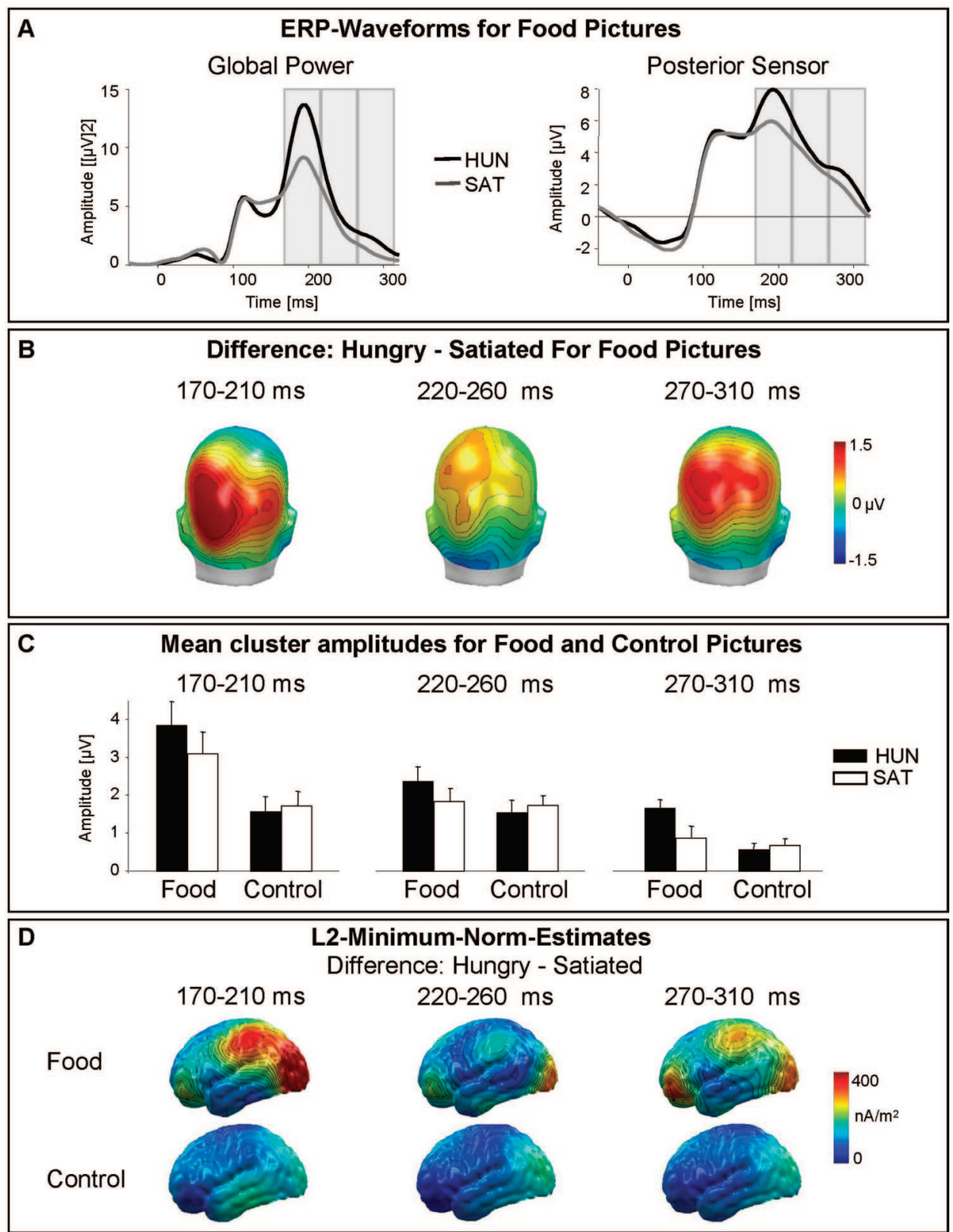

Figure 1. (A) Event-related brain potential (ERP) waveforms for food pictures in hungry (HUN) and satiated (SAT) states. The left side illustrates the global field power (i.e., the mean of the squared potentials across all electrodes) serving as a global measure of scalp potentials. The right side shows the waveform of a representative posterior sensor (\#66). (B) Scalp potential maps show the topography of the difference between hungry and satiated states for food-cue processing in three time intervals from 170 to $310 \mathrm{~ms}$. Illustrated is the back view of a model head. Voltages were interpolated to the scalp surface with spherical splines. (C) Mean amplitudes of selected posterior sensor clusters (averages across left and right), showing differences between hungry (HUN) and satiated (SAT) states for food and nonfood control pictures, averaged across pleasant, neutral, and unpleasant picture contents. (D) L2 minimum-norm estimates of the deprivation effects for food and nonfood control pictures for the three time intervals.

\section{L2 MN Analysis}

As is shown in Figure 1D, the difference in processing food cues in a deprived state in comparison with a satiated state was primarily modeled by sources over occipito-temporo-parietal regions. Moreover, L2 MN solutions indicated secondary sources over frontal regions, suggesting that the polarity reversal of the deprivation effect over anterior sites may not solely reflect polarity reversal but, at least in part, engagement of 
anterior brain regions. In contrast, L2 MN solutions for nonfood control pictures showed no systematic differences as a function of the food deprivation.

\section{Replication of Selective Emotion Processing: Early Posterior Negativity}

Previous studies consistently observed that emotional rather than neutral picture processing is associated with an increased early posterior negative (EPN) potential, most pronounced between 200 and $300 \mathrm{~ms}$ poststimulus (Schupp et al., 2007). To pursue these findings, we explored whether the EPN component is affected by food deprivation. By replicating previous findings, we observed a significant main effect of Valence, $F(2,30)=63.9, p=.001$. Pleasant and unpleasant pictures elicited a more pronounced posterior negativity in comparison with neutral pictures, $F \mathrm{~s}(1,15)=102.2$ and $71.4, p<$ .001 , respectively. Furthermore, this analysis indicated neither a differential influence of the motivational state on emotional picture processing, State $\times$ Valence, $F(2,30)<1$, nor a significant main effect of State, $F(1,15)=3.0, p=.11$.

\section{Discussion}

This study explores whether the perceptual processing of food pictures is modulated by the current motivational state. In support of this hypothesis, we observed a significant deprivation effect over posterior sensor clusters contrasting the processing of food cues in hungry and satiated states. The results revealed that deprivation effects were limited to food cues and did not extend to the processing of nonfood appetitive or aversive stimuli. The novel finding of this study is that internal motivational factors already modulate the perception of food stimuli around $170 \mathrm{~ms}$ poststimulus. Such a state-dependent increase in the salience of food cues may be an important mechanism to efficiently organize foodrelated behaviors (LaBar et al., 2001; Lang et al., 1997; Öhman et al., 2000).

A number of recent Positron Emission Tomography (PET)- and fMRI studies suggest that food deprivation and food stimulus processing affect the activity in limbic structures (amygdala, orbitofrontal gyrus, and insular cortex) as well as the extrastriate visual cortex (Cheng, Meltzoff, \& Decety, 2007; Killgore et al., 2003; LaBar et al., 2001; Morris \& Dolan, 2001; Simmons, Martin, \& Barsalou, 2005; Porubská, Veit, Preissl, Fritsche, \& Birbaumer, 2006; Uher, Treasure, Heining, Brammer, \& Campbell, 2006). Most relevant to this study, LaBar et al. (2001) observed that food deprivation enhanced the activity in higher-order visualassociative regions and the amygdala when processing food pictures, considered to reflect the enhancement of visual attention to the need-related cues. The finding that hunger increased visual attention to food cues was followed up by this study, emphasizing the temporal dynamics of these effects. Confirming the selective processing of food pictures, ERPs revealed enhanced posterior positive deflections for food pictures in a hungry versus a satiated state, developing already around $170 \mathrm{~ms}$ and lasting for approximately $150 \mathrm{~ms}$. Enhancing the spatial resolution of surfacerecorded brain potentials by $\mathrm{L} 2 \mathrm{MN}$ source calculations, deprivation effects on food processing were associated with primary activations in posterior perceptual representation networks. In addition, secondary activations were suggested over anterior brain regions. However, spatial resolution is insufficient to determine whether this finding relates to orbitofrontal activations, which were observed when comparing food-cue processing in hungry and satiated states and food stimuli varying in their incentive value (Arana et al., 2003; Critchley \& Rolls, 1996; Gottfried, O'Doherty, \& Dolan, 2003; Morris \& Dolan, 2001; O'Doherty et al., 2000). Furthermore, deprivation effects appeared more pronounced over left-hemispheric regions. Recent fMRI studies observed that the activation of the amygdala in the context of food-cue processing is more pronounced in the left amygdala (LaBar et al., 2001; Morris \& Dolan, 2001). Considering the hypothesis that perceptual processing at the cortical level is regulated by activity in subcortical limbic structures (cf. Vuilleumier, 2005), one may speculate whether lateralized ERP findings reflect effective connectivity of cortico-subcortical structures. Overall, consistent with the hypothesis of the state-dependent motivational regulation of selective attention, the present findings demonstrate that hunger modulates the processing of food cues early in the visual processing stream.

An important feature of the present design was to include emotionally significant control materials to determine the specificity of food-deprivation effects (Drobes et al., 2001; Lüthy et al., 2003; Mauler et al., 2006). Consistent with previous research, deprivation specifically affected subjective evaluation of food pictures. With regard to enhanced positive potentials for food cues over posterior regions, deprivation had no effect on appetitive, neutral, or aversive control materials. Furthermore, the finding that the emotion-sensitive EPN component did not vary as a function of hunger state also suggests that food deprivation did not elicit general arousal effects (cf. Hull, 1943). Thus, regarding the processing of emotional control pictures, no evidence was observed for the interaction of the two routes of motivational influence, that is, the inherent significance of the stimulus and the current motivational state. The present findings are furthermore consistent with research demonstrating that food deprivation specifically enhanced the reinforcing value of food cues and approach tendencies toward food cues (Epstein \& Leddy, 2006; Seibt, Häfner, \& Deutsch, 2007). All told, this study obtained evidence that food-deprivation effects are specific to the processing of food cues.

While the latency of the deprivation effect was similar as in previous studies that examined emotionally significant cues or explicit feature-based selective attention, the effects appear different with regard to their topographical attributes (cf. Codispoti, Ferrari, Junghöfer, \& Schupp, 2006; Junghöfer, Bradley, Elbert, \& Lang, 2001). Specifically, the difference potential associated with stimulus significance is characterized by a broadly distributed negativity over occipitotemporal areas and a positive difference over central sites. In comparison, the deprivation effect observed here revealed small negative deflections over inferior occipital areas and a predominant positive difference over parieto-occipital sites (see Figure 1B). While the present data do not allow us to know whether these differences reflect a reversal in polarity or reveal a shift in topography, several aspects may contribute to these differences. Food stimuli may elicit activity in distinct higher-order visual-associative regions, as has been reported for faces, human bodies, tools, and locations (reviewed by Malach, Levy, \& Hasson, 2002). Thus, in comparison with the topography of selective visual attention effects associated with emotion and target processing, differences in scalp potential fields associated with need-related changes in food processing may reflect differ- 
ences in the engagement of food-selective visual-associative brain regions, differences in dipole orientation, or both. Interestingly, L2 MN source analysis revealed increased activation over posterior perceptual and anterior regions for the cue-specific deprivation effect, concurring with neural source analysis reported for emotional and target stimulus processing (cf. Codispoti et al., 2006; Junghöfer et al., 2001, 2006). Thus, while the data are consistent with the notion of a state-dependent regulation of perception and visual attention, more research is needed to demonstrate that both the state-dependent motivational and the emotional regulation of visual attention are based on similar neural mechanisms.

Some limitations with regard to this study need to be considered. Compliance with the instruction was measured by means of selfreport and a "bogus pipeline" urine sample (cf. Drobes et al., 2001). However, subjective ratings of hunger pronouncedly increased (Drobes et al., 2001; Leland \& Pineda, 2006; Mogg et al., 1998), and as in previous research (Lüthy et al., 2003; Mauler et al., 2006), food stimuli were judged more pleasant and more arousing after deprivation than in a satiated state. Thus, while no physiological data were obtained as a manipulation check, subjective report suggests that participants complied with the eating protocol. Furthermore, only men were examined. As recent studies have shown gender differences in the context of food deprivation (Stoeckel, Cox, Cook, \& Weller, 2007; Uher et al., 2006), the selective processing of food cues in a hungry state needs to be further explored in women.

In conclusion, this study provides evidence for the notion that hunger modifies the perceptual processing of food cues. Fooddeprivation effects were specific to food stimuli and did not affect the perceptual processing of pleasant, neutral, and unpleasant natural scenes taken from the IAPS library. Considered from the perspective of motivated attention (Lang et al., 1997), the statedependent increase in the salience of food-related visual cues may foster behaviors needed to restore energy levels.

\section{References}

Arana, F. S., Parkinson, J. A., Hinton, E., Holland, A. J., Owen, A. M., \& Roberts, A. C. (2003). Dissociable contributions of the human amygdala and orbitofrontal cortex to incentive motivation and goal selection. Journal of Neuroscience, 23, 9632-9638.

Bradley, M. M., \& Lang, P. J. (1994). Measuring emotion: The SelfAssessment Manikin and the Semantic Differential. Journal of Behavior Therapy and Experimental Psychiatry, 25, 49-59.

Bradley, M. M., Sabatinelli, D., Lang, P. J., Fitzsimmons, J. R., King, W., \& Desai, P. (2003). Activation of the visual cortex in motivated attention. Behavioral Neuroscience, 117, 369-380.

Channon, S., \& Hayward, A. (1990). The effect of short-term fasting on processing of food cues in normal subjects. International Journal of Eating Disorders, 9, 447-452.

Cheng, Y., Meltzoff, A. N., \& Decety, J. (2007). Motivation modulates the activity of the human mirror-neuron system. Cerebral Cortex, 17, 1979-1986.

Codispoti, M., Ferrari, V., Junghöfer, M., \& Schupp, H. T. (2006). The categorization of natural scenes: Brain attention networks revealed by dense sensor ERPs. Neuroimage, 32, 583-591.

Critchley, H. D., \& Rolls, E. T. (1996). Hunger and satiety modify the responses of olfactory and visual neurons in the primate orbitofrontal cortex. Journal of Neurophysiology, 75, 1673-1686.

Derryberry, D., \& Tucker, D. M. (1994). Motivating the focus of attention. In P. M. Niedenthal \& S. Kitayama (Eds.), The heart's eye: Emotional influences in perception and attention (pp. 167-196). San Diego, CA: Academic Press.

Diehl, J. M., \& Staufenbiel, T. (1994). Inventar zum Essverhalten und Gewichtsproblemen (IEG). Frankfurt, Germany: Klotz.

Dobson, K. S., \& Dozois, D. J. (2004). Attentional biases in eating disorders: A meta-analytic review of Stroop performance. Clinical Psychology Review, 23, 1001-1022.

Drobes, D. J., Miller, E. J., Hillman, C. H., Bradley, M. M., Cuthbert, B. N., \& Lang, P. J. (2001). Food deprivation and emotional reactions to food cues: Implications for eating disorders. Biological Psychology, 57, 153-177.

Epstein, L. H., \& Leddy, J. J. (2006). Food reinforcement. Appetite, 46, $22-25$.

Gottfried, J. A., O’Doherty, J., \& Dolan, R. J. (2003). Encoding predictive reward value in human amygdala and orbitofrontal cortex. Science, 301, $1104-1107$.

Hauk, O. (2004). Keep it simple: A case for using classical minimum norm estimation in the analysis of EEG and MEG data. NeuroImage, 21 $1612-1621$

Hull, C. L. (1943). Principles of behavior. New York: Appleton.

Junghöfer, M., Bradley, M. M., Elbert, T. R., \& Lang, P. J. (2001). Fleeting images: A new look at early emotion discrimination. Psychophysiology, $38,175-178$.

Junghöfer, M., Elbert, T., Tucker, D. M., \& Rockstroh, B. (2000). Statistical control of artifacts in dense array EEG/MEG studies. Psychophys iology, 37, 523-532.

Junghöfer, M., \& Peyk, P. (2004). Analyse und Visualisierung von Hirnstrom- und Hirnmagnetfeld-Messung. Matlab Select, 2, 24-28.

Junghöfer, M., Sabatinelli, D., Bradley, M. M., Schupp, H. T., Elbert, T. R., \& Lang, P. J. (2006). Fleeting images: Rapid affect discrimination in the visual cortex. Neuroreport, 17, 225-229.

Killgore, W. D , Young, A. D., Femia, L. A., Bogorodzki, P., Rogowska, J., \& Yurgelun-Todd, D. A. (2003). Cortical and limbic activation during viewing of high- versus low-calorie foods. NeuroImage, 19, 1381-1394.

LaBar, K. S., Gitelman, D. R., Parrish, T. B., Kim, Y. H., Nobre, A. C., \& Mesulam, M. M. (2001). Hunger selectively modulates corticolimbic activation to food stimuli in humans. Behavioral Neuroscience, 115 493-500.

Lang, P. J., Bradley, M. M., \& Cuthbert, B. N. (1997). Motivated attention: Affect, activation, and action. In P. J. Lang, R. F. Simons, \& M. Balaban (Eds.), Attention and emotion: Sensory and motivational processes (pp. 97-135). Mahwah, NJ: Erlbaum.

Lang, P. J., Bradley, M. M., \& Cuthbert, B. N. (2005). International affective picture system (IAPS): Digitized photographs, instruction manual and affective ratings. Tech. Rep. No. A-6. Gainesville, FL: University of Florida.

Lavy, E. H., \& van den Hout, M. A. (1993). Attentional bias for appetitive cues: Effects of fasting in normal subjects. Behavioural and Cognitive Psychotherapy, 21(4), 297-310.

Leland, D. S., \& Pineda, J. A. (2006). Effects of food-related stimuli on visual spatial attention in fasting and nonfasting normal subjects: Behavior and electrophysiology. Clinical Neurophysiology, 117, 67-84.

Luck, S. J., Woodman, G. F., \& Vogel, E. K. (2000). Event-related potential studies of attention. Trends in Cognitive Sciences, 4, 432-440.

Lüthy, M., Blumenthal, T. D., Langewitz, W., Kiss, A., Keller, U., \& Schächinger, H. (2003). Prepulse inhibition of the human startle eye blink response by visual food cues. Appetite, 41, 191-195.

Malach, R., Levy, I., \& Hasson, U. (2002). The topography of high-order human object areas. Trends in Cognitive Sciences, 6, 176-184.

Margraf, J., Schneider, S., \& Ehlers, A. (1991). Diagnostisches interview bei psychischen Störungen: DIPS (Diagnostical interview for mental disorders: DIPS). Berlin, Germany: Springer.

Mauler, B. I., Hamm, A. O., Weike, A. I., \& Tuschen-Caffier, B. (2006). Affect regulation and food intake in bulimia nervosa: Emotional re- 
sponding to food cues after deprivation and subsequent eating. Journal of Abnormal Psychology, 115, 567-579.

Mogg, K., Bradley, B. P., Hyare, H., \& Lee, S. (1998). Selective attention to food-related stimuli in hunger: Are attentional biases specific to emotional and psychopathological states, or are they also found in normal drive states? Behaviour Research and Therapy, 36, 227-237.

Morris, J. S., \& Dolan, R. J. (2001). Involvement of human amygdala and orbitofrontal cortex in hunger-enhanced memory for food stimuli. Journal of Neuroscience, 21, 5304-5310.

O’Doherty, J., Rolls, E. T., Francis, S., Bowtell, R., McGlone, F., Kobal, G., Renner, B., \& Ahne, G. (2000). Sensory-specific satiety-related olfactory activation of the human orbitofrontal cortex. Neuroreport, 11, 893-897.

Öhman, A., Flykt, A., \& Lundqvist, D. (2000). Unconscious emotion: Evolutionary perspectives, psychophysiological data and neuropsychological mechanisms. In R. D. Lane \& L. Nadel (Eds), Cognitive neuroscience of emotion (pp. 296-327). New York: Oxford University Press.

Placanica, J. L., Faunce, G. J., \& Soames Job, R. F. (2002). The effect of fasting on attentional biases for food and body shape/weight words in high and low Eating Disorder Inventory scorers. International Journal of Eating Disorders, 32, 79-90.

Porubská, K., Veit, R., Preissl, H., Fritsche, A., \& Birbaumer, N. (2006). Subjective feeling of appetite modulates brain activity: An fMRI study. NeuroImage, 32, 1273-1280.

Sabatinelli, D., Bradley, M. M., Fitzsimmons, J. R., \& Lang, P. J. (2005). Parallel amygdala and inferotemporal activation reflect emotional intensity and fear relevance. NeuroImage, 24, 1265-1270.

Sabbagh, M. A., \& Taylor, M. (2000). Neural correlates of theory-of-mind reasoning: An event-related potential study. Psychological Science, 11, $46-50$.

Schupp, H. T., Junghöfer, M., Weike, A. I., \& Hamm, A. O. (2003).
Emotional facilitation of sensory processing in the visual cortex. Psychological Science, 14, 7-13.

Schupp, H. T., Stockburger, J., Codispoti, M., Junghöfer, M., Weike, A. I., \& Hamm, A. O. (2006). Stimulus novelty and emotion perception: The near absence of habituation in the visual cortex. Neuroreport, 17, 365 369.

Schupp, H. T., Stockburger, J., Codispoti, M., Junghöfer, M., Weike, A. I., $\&$ Hamm, A. O. (2007). Selective visual attention to emotion. Journal of Neuroscience, 27, 1082-1089.

Seibt, B , Häfner, M \& \& Deutsch, R. (2007). Prepared to eat: How immediate affective and motivational responses to food cues are influenced by food deprivation. European Journal of Social Psychology, 37, 359-379.

Simmons, W. K., Martin, A., \& Barsalou, L. W. (2005). Pictures of appetizing foods activate gustatory cortices for taste and reward. Cerebral Cortex, 15, 1602-1608.

Stoeckel, L. E., Cox, J. E., Cook III, E. W., \& Weller, R. E. (2007). Motivational state modulates the hedonic value of food images differently in men and women. Appetite, 48, 139-144.

Stunkard, A. J., \& Messick, S. (1985). The three-factor eating questionnaire to measure dietary restraint, disinhibition and hunger. Journal of Psychosomatic Research, 29, 71-83.

Uher, R., Treasure, J., Heining, M., Brammer, M. J., \& Campbell, I. C. (2006). Cerebral processing of food-related stimuli: Effects of fasting and gender. Behavioural Brain Research, 169, 111-119.

Vuilleumier, P. (2005). How brains beware: Neural mechanisms of emotional attention. Trends in Cognitive Sciences, 9, 585-594. 